\title{
Integrating Computational Design to Improve the Design Workflow of Modular Construction
}

\author{
Tom Greenough ${ }^{1 *}$, Matthew Smith ${ }^{2}$, and Aaron Mariash ${ }^{3}$ \\ ${ }^{1}$ Associate, Entuitive \\ ${ }^{2}$ Senior Engineer, Entuitive \\ ${ }^{3}$ Technologist, Entuitive \\ ${ }^{*}$ Corresponding author's e-mail: tom.greenough@entuitive.com
}

\begin{abstract}
The construction industry's productivity has stagnated since the 1960s while in the same period, manufacturing and technology industries have seen vast improvements. The construction industry is coming under increased pressure to provide better value through improved quality and performance and as a result developers and constructors are looking to alternative forms of construction. In a process that borrows concepts from the manufacturing and technology sectors, such as automation and 3D modelling, Prefabricated Volumetric Construction (modular construction) is a highly versatile approach with the potential to deliver substantial cost savings, faster project delivery times, higher quality construction with less waste and emissions, and an increase in worker safety. This paper will explore the integration of computational design into the design workflow of modular construction through several project examples. The following topics will be covered: creation and assembly of parametric modules in the Revit Building Information Model (BIM) software, using the visual programming tool Dynamo; the linkage of the BIM to the analytical model; and the extraction of results and the manipulation and display of data using Grasshopper, a visual programming language and environment plugin, for Rhinoceros a 3D modeler. This integration has been found to reduce the time required to develop the building model, the drawings, the analytical model and complete the design while improving the consistency and accuracy of all.
\end{abstract}

\section{KEYWORDS}

Prefabricated Volumetric Construction; Modular construction; Computational design; Building Information Model; Design

\section{INTRODUCTION}

Productivity is defined as the measure of the rate at which work is performed. It is the ratio of production output to what is required to produce it. The construction industry's productivity has stagnated since the 1960s while in the same period, manufacturing and technology industries have seen vast improvements (McKinsey Global Institute, 2017). In the context of Prefabricated Volumetric Construction (PVC), the output can be viewed as the number of modules designed and produced and the input resource is person-hours. The productivity of the construction industry continues to lag for a multitude of factors including the following (Farmer, 2016; Fuchs, 2018): 
- An overall sector organization that is siloed.

- A design process that views the development of each project as a one-of-a kind product.

- Underinvestment in technology and innovation across the industry.

- Contract structures and procurement protocols that result in a more adversarial environment rather than a collaborative one.

- A design-construct-supply chain that is heavily discretized and fragmentated.

Combine this with the vulnerability associated with a shrinking skilled labour force (Modular Building Institute, 2018) and it is time for the industry to decide to "Modernise or Die" (Farmer, 2016). The National Research Council (NRC) formed an ad-hoc committee in 2009 to search for solutions to this productivity crisis. This committee identified five breakthroughs as having the most significant impact on the productivity of the construction sector which included widespread use of Building Information Modelling (BIM) and interoperable technology applications; and the use of prefabrication, preassembly, modularization, and off-site fabrication techniques and processes (Modular Building Institute 2010). To expose inefficiencies and boost productivity the industry needs to adopt a more manufacturing approach with mass production and standardization with repeatable design elements that reduce the number of bespoke solutions required. The use of technology can further augment this from accelerating the development and accuracy of the design and design documents to enabling visualization of problem areas and improving construction planning.

This paper will explore the integration of computational design into the design workflow of modular construction through several project examples. The examples illustrated here include Cheatham Street Flats (CSF), a five-storey, 140-suite (323 modules) student residence in Texas (Figure 1); and CORE Nolensville (CORE), a five-storey 125-suite (270-modules) condo development in Tennessee. The chassis of the modules is of structural steel construction as shown in Figure 2. The floor and ceiling framing along with the columns and braces are hollow structural steel sections (HSS). The floor and ceiling framing are connected to the columns using a propriety cast-steel connector (supplied by Z Modular, a division of Zekelman Industries, and trademarked as Vectorbloc). Once the individual modules are erected on site, they are linked horizontally and vertically by way of steel gusset plates bolted to the modules.
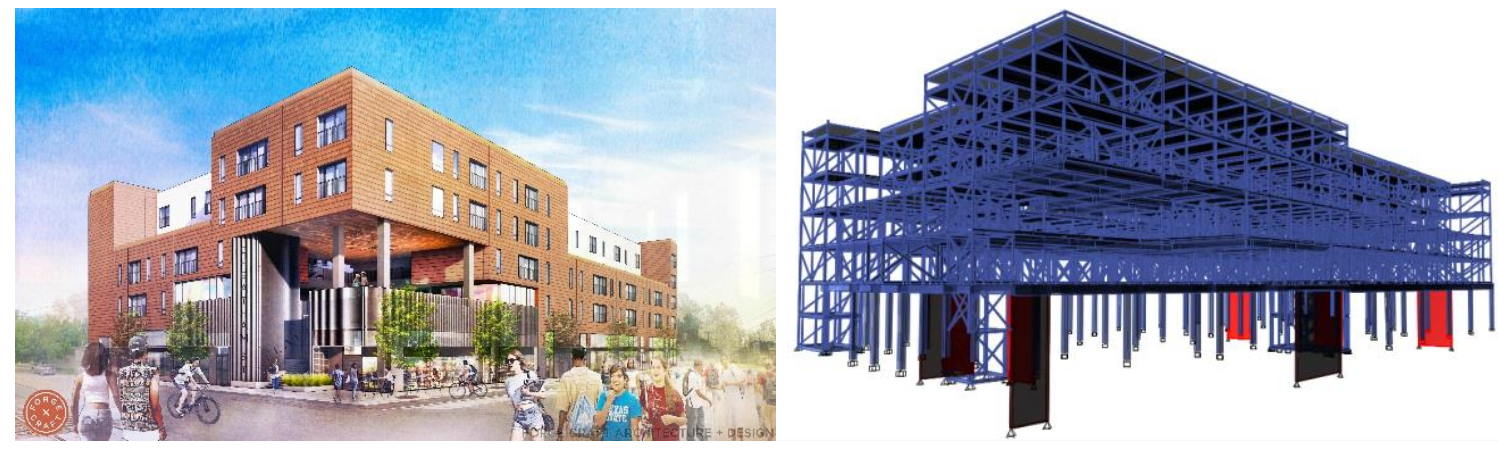

Figure 1. CSF, architectural rendering (left) and corresponding analytical model (right). Rendering courtesy of Forge Craft Architecture. 


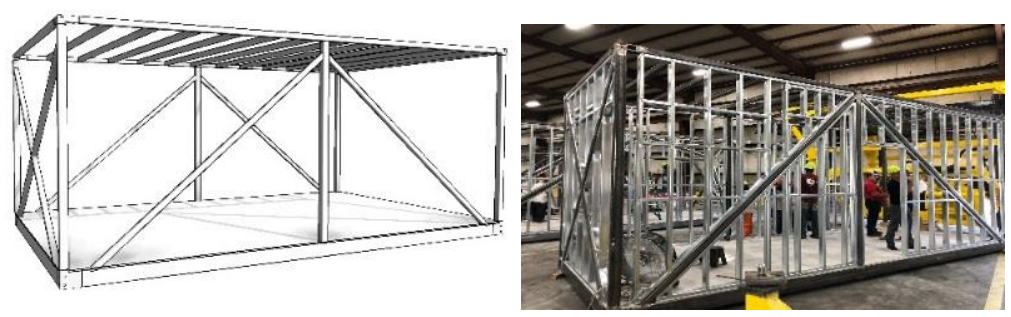

Figure 2. Modular chassis, BIM (left) and as fabricated (right).

Computational design is the application of programming to the design process including but not limited to design generation and task automation. Most computational design environments rely on a visual programming interface to assemble the code graphically rather than traditional textbased script programming. In building design, Grasshopper and Dynamo are the most well-known graphical algorithm editors. Grasshopper is a visual programming plugin for Rhinoceros, the 3D modelling software developed by Robert McNeel and Associates. Dynamo is a visual programming plugin for Autodesk's Revit. Computational design has made a name for itself in the modelling of complex geometry, but these tools can also be used where there is a lot of repetition in the building or in the automation of tedious tasks. In this paper the following topics will be covered: creation and assembly of parametric modules in Revit using Dynamo; the linkage of the resulting BIM to the analytical model; and the extraction of results and the manipulation and display of data using Grasshopper and Rhino. While adoption of computation design in the Architectural Engineering Construction (AEC) industry is well underway (Lattmann, 2018), the authors have found limited published examples of computational design in the prefabrication, offsite construction and modular sectors, suggesting this is currently a nascent application (Doe, 2018; Epp, 2018).

\section{DYNAMO PARAMETRIC MODELLING IN REVIT}

A useful approach to creating adaptable models for modular construction projects is the integration of automation into the early stages of the design process. In the case of CORE, the process involved the development of a Dynamo script which assembles the chassis framework given a set of parametric inputs. The script is divided into two steps: geometry creation/modification, and structural member assignment. The first half of the script establishes the basic geometry of the chassis using known and assumed requirements - overall chassis length, width, and height, number and the spacing of floor and ceiling joists. Each of these parameters can be selected and modified using a series of "sliders". The overall dimension parameters impact the location of intermediate support columns and the arrangement of vertical bracing members. Finally, individual offsets for the location of the ends of vertical bracing members can be modified to accommodate standard or unique detailing requirements. Once the geometric parameters have been assigned, a wireframe skeleton is created (Figure 3). In the second half of the script a series of dropdown menus created in Dynamo insert Revit components, giving the user the ability to quickly assign individual member sizes to floor and ceiling beams and joists, vertical bracing, exterior columns, intermediate columns, and floor types. In addition, the script also includes location and selection options for the proprietary cast steel Vectorbloc connectors, which are present at upper and lower corners, and at certain interior columns when necessary. 


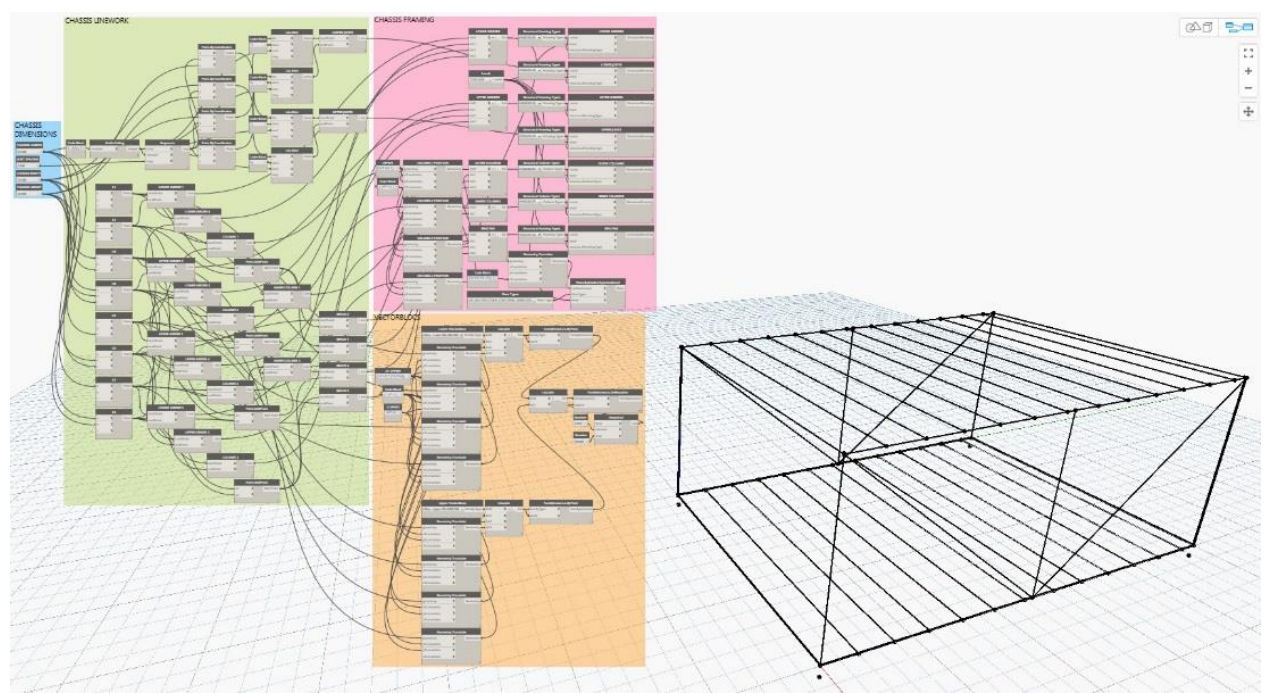

Figure 3. Dynamo script (left) and resulting wireframe chassis (right).

In addition to creating the modelled elements, the script also generates Revit's analytical data, represented by lines and nodes, which will be useful later in the process (Figure 4). Arrangement of the geometry and framing selection takes only a few seconds, and depending on computer performance, generating the model in Revit takes less than a minute more. For efficiency, it is useful to create a Revit Template file which contains a selection of Revit components that may be required to frame each suite, along with the level datum for the module floor and the module ceiling height.

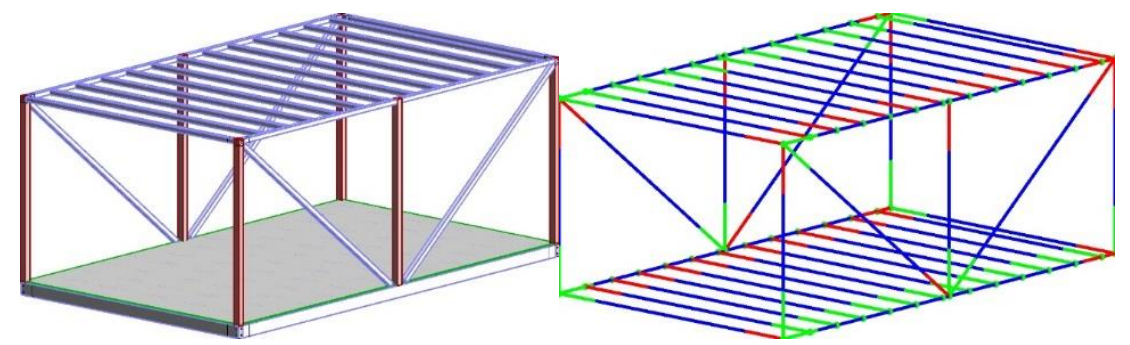

Figure 4. Model and analytical view of chassis framing for individual module.

To assemble a complete building, the Dynamo script generates individual chassis similar to the one pictured above, which can be combined into more complicated arrangements for suites of various sizes and complexity. In the case of CORE, five suite types were created consisting of one to three unique chassis arrangements each and along with the elevator, stair, and common area modules a total of 12 unique chassis were required for the 270-module project. Individual Revit Project Files were created for each type of suite and Dynamo was used to generate the framing for the required chassis in each file. In some cases, after running the script and assembling the chassis for each suite, some adjustment of framing cutback lengths and analytical connections were required.

Once each of the Revit Project Files were assembled, they were linked into a master coordination model, along with the architectural model to guide the placement of each suite. By using a series 
of linked Revit Project Files, arrangement of the units becomes a simple drag and drop operation. The geometry contained in each Project File can be manipulated as "blocks" without compromising the suite in any way (Figure 5). Using this technique, creating the fully assembled framework of a large and complicated building can be accomplished in short order.

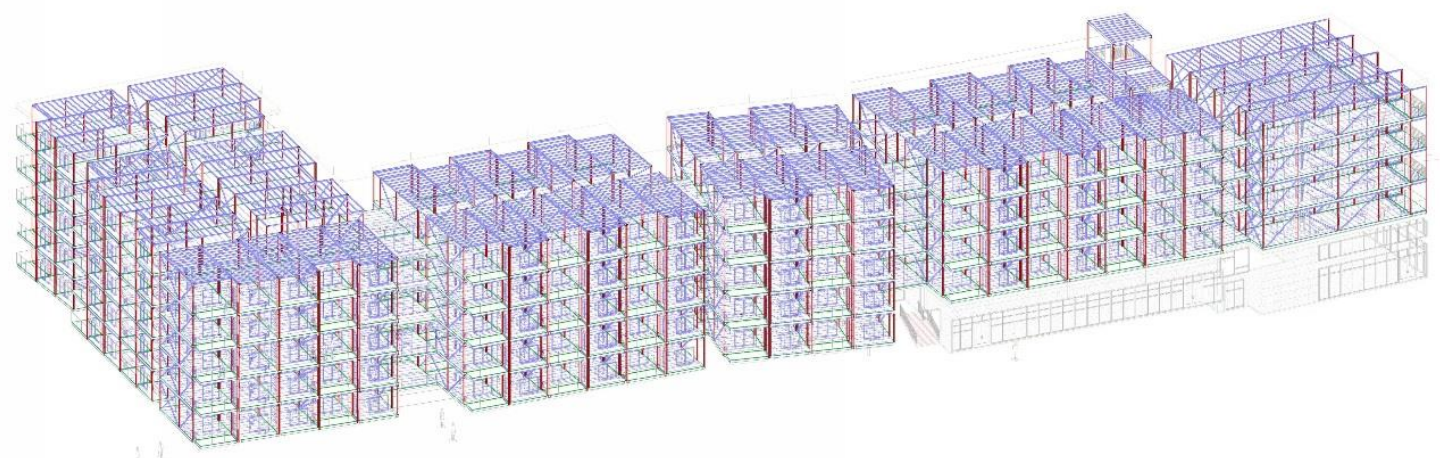

Figure 5. Assembled chassis modules into master model.

\section{LINKING BIM TO ANALYTICAL MODELS}

The current workflow for many consulting firms is to use one software program to create the Contract Documents, such as AutoCAD or Revit, and another or several other software programs to create the analytical models and complete the design. This results in duplication of effort, inefficiencies and higher chances for error. With the ability to now rapidly generate a BIM using computational design, productivity can be further enhanced by linking it to analytical programs such as SAP and ETABS which are developed by Computers and Structures Inc.

Along with the geometry, the master model from the previous step contains the analytical data for each module chassis (Figure 6). Revit's analytical tools can be temperamental when working with complex geometry. Using individual Revit Project Files to adjust analytical framing and connection information reduces the time required to check through an entire structural model and reliably maintains the analytical geometry and connection of the nodes in a chassis when arranging the master layout. Once the master layout has been completed, it is possible to link Revit's analytical data to an analytical program, in this case SAP (Figure 7), to complete the structural analysis and design. After the initial model is set, there are often decisions that impact or change the design. These changes often need to be made in both the BIM software and the analytical software. In this process a change as drastic as flipping the building to suit site grading can be made rapidly in Revit and a new analytical model created.

Once the analytical model has been exported to the chosen structural analysis program, design engineers complete the analysis model by defining gravity and lateral loads, as well as load combinations. Current studies are looking at how much of this additional information required for the design can also be captured in Dynamo to reduce time in updating analytical models after a revision. With loading and load combinations defined, engineers can run the analysis and complete the design of the building using the resulting forces and deformations. 


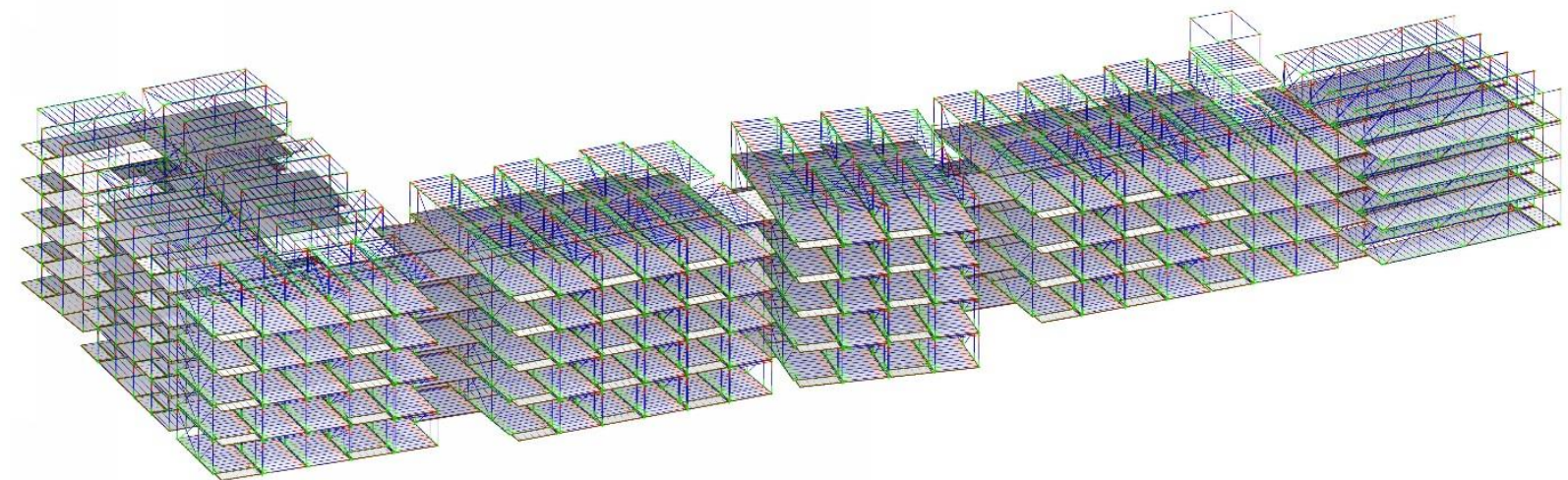

Figure 6. Complete building analytic model in Revit.

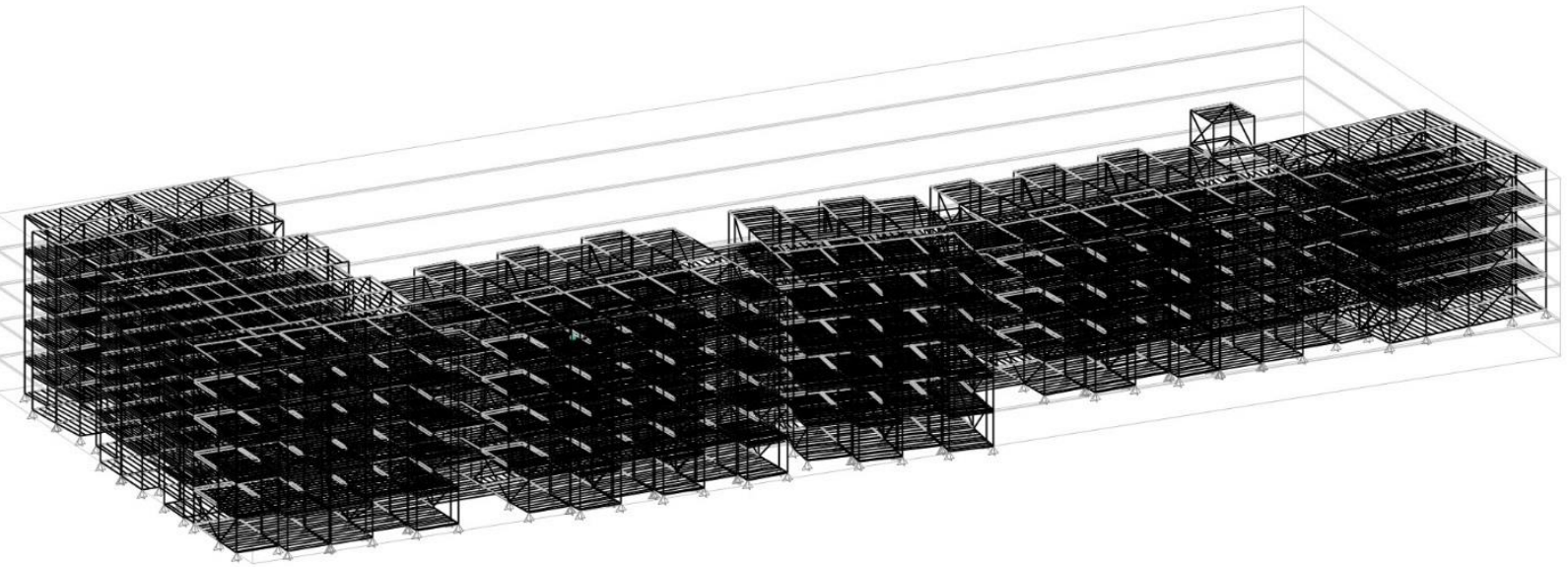

Figure 7. Complete building analytic model in SAP.

\section{DATA MANAGEMENT}

In an ideal scenario, all the modelling and analysis would be completed and documented in one software package. However, modular structures contain in their analysis models a quantity of elements that is an order of magnitude greater than a conventional structure of similar size due to the spacing of members and the modelling of structural connections between modules. This creates challenges for using and displaying the results of the analysis in an efficient and accurate manner, which is done either graphically or in tabular form in the native analytical programs. Here, computational design tools are again leveraged to manage the mass amount of data that is generated. This improves efficiency and errors are reduced.

Within PVC, multiple modules may be supported on a single foundation element at their base. From the analysis model, this would appear as multiple reactions that must be summed for all load combinations considered. This process has been automated in Grasshopper and is shown in Figure 8. The script, written in the coding language Python within Grasshopper, locates all reactions in space using their cartesian coordinates, determines if they should be summed or not depending on their spatial proximity, and then sums all components of their reactions for each load combination. This has been found to reduce the amount of time spent by engineers to group, size, and design foundation elements by up to $50 \%$. 

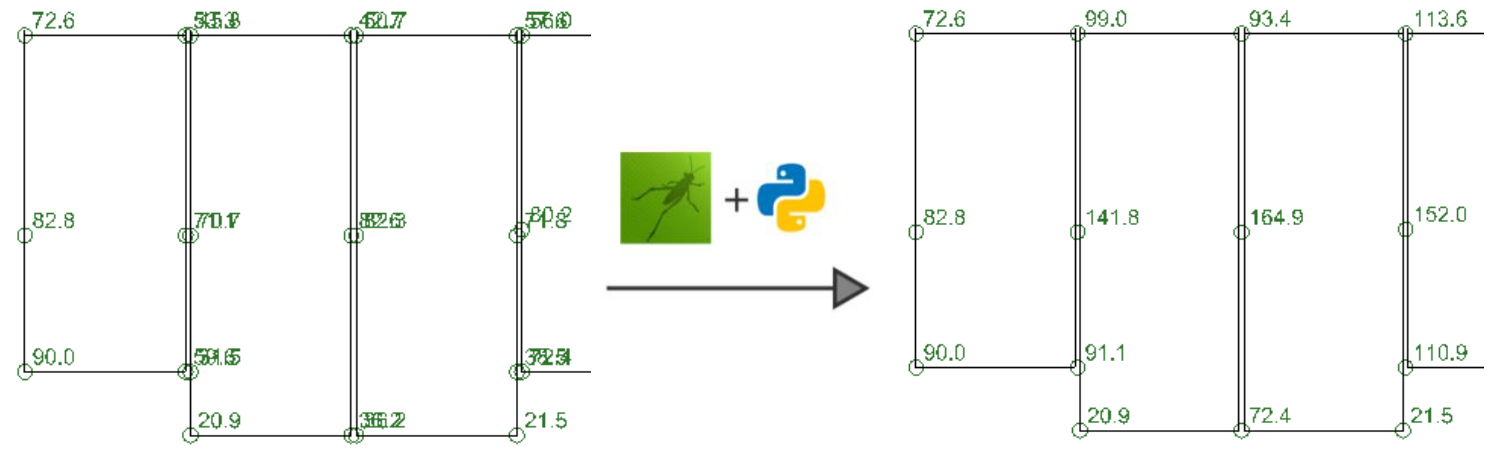

Figure 8. Vertical base reactions of CORE, original (left) and post-processed (right).

In addition to post-processing data for calculations and design checks, computational design tools can be used to identify areas within the structure that need specific attention. On CSF, for example, multiple modules within the structure were experiencing net uplift in their vertical connections. These locations had to be identified in the Contract Documents so that connections could be pretensioned, however over-specifying the number of pretensioned locations would introduce unnecessary inefficiencies in the erection process. Data from the analysis was exported into Grasshopper, where locations of net uplift were located within the structure and affected modules were tagged accordingly. This took what would be a very time-consuming task in the native analytical program and automated it, resulting in a time savings of $80 \%$ to $90 \%$.

Lastly, using computational design tools for data management can allow for summarizing data in a more concise and intuitive manner than is possible within the native analytical programs. An example of this is on CSF, where almost every module has bracing and there is a transfer structure at Level 3 and Level 4. This creates a very complex load path for lateral forces and tracking these lateral load paths in the native analytical programs can be difficult due to the large quantity of elements. By summarizing axial brace forces in Grasshopper and projecting them onto 2D floor plans, engineers were able to follow the load path in an intuitive manner and ensure the structure would perform adequately in extreme winds or earthquakes. This also identified areas in the diaphragm that required special attention since large forces were being transferred from one bracing line to another between floors. An example of these brace force floor plans automated from Grasshopper is shown in Figure 9.

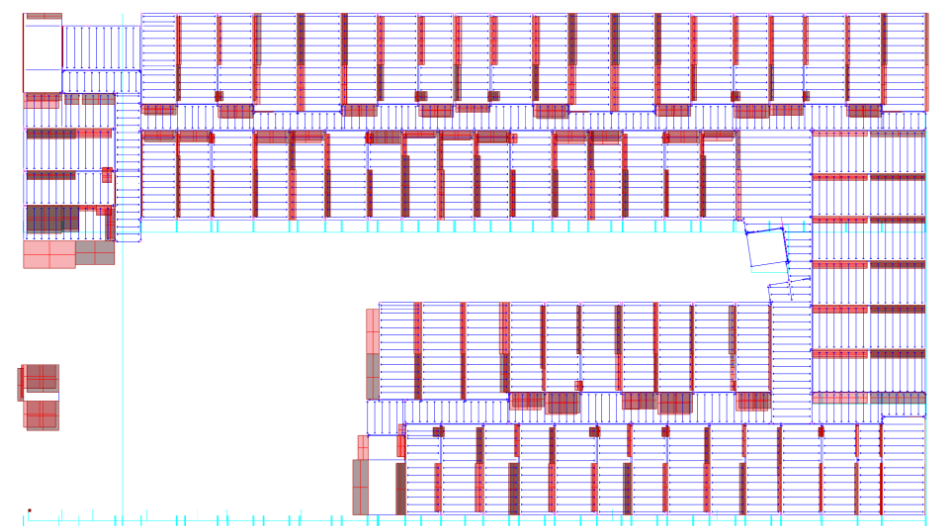

Figure 9. Brace Force Distribution. 


\section{CONCLUSION}

Innovation is everywhere and for the modular construction industry the opportunities to reduce project complexity, raise productivity and value are significant. Rapid advances in computational design and the ability to utilise data create opportunities to revolutionise the process of design, engineering and construction. But technological advancement without practical experience can create solutions that don't deliver or integrate. This paper has explored several computational design tools created to solve real life engineering challenges. The tools have shown great promise and reduced the time required to accurately model modular buildings containing thousands of steel elements. The tools reduced process fragmentation, optimized processes, improved data accuracy and reduce the time spent on data entry. Furthermore, the processes identified in this paper will significantly improve the overall quality of the design process, reducing issues downstream by affording the opportunity to model and analyse multiple design options faster and earlier in the design process. In short, the tools described in this paper can have a large impact on the productivity of the design workflow and can revolutionize the way we model, design and engineer.

Future evolution and research opportunities exist for these workflow tools including the following:

- Exploration of other plug-ins and analysis programs. The primary challenge to the overall efficiency of the current workflow in Revit 2018 comes in the binding and adjustment of analytical data in the master model. Experiments in Revit 2019 have shown some improvements to model group integrity and stability as well as interoperability between linked Revit Project Files, but the issue is not fully resolved.

- Loading into Grasshopper the capacity of the fabricator preferred members and standard connection details and developing scripts to identify where specific elements or connections exceed this capacity and flag for special attention by engineers.

- Linkage to the fabricators shop drawing production tool and procurement database.

\section{REFERENCES}

Doe, R. (2018). "Facilitating integration of computational design processes in the design and production of prefabricated homes." Architectural Science Review, 61:4, 246-254.

Epp, L. (2018). “Computational Design with Timber.” Wood Design \& Buildings, Winter 201718 No.78, 39-43.

Farmer, M. (2016). "The Farmer Review of The UK Construction Labour Model." Construction Leadership Council.

Fuchs, S. (2018). "Reinventing Construction: A Route to Higher Productivity." Modular Advantage, 2018Q1, 10-13.

Lattmann, G. (2018). “10 Years of CanBIM.” Innovation Spotlight 2018, CanadaBIM Council.

McKinsey Global Institute (2017). "Reinventing Construction: A Route to Higher Productivity." $<$ https://www.mckinsey.com/industries/capital-projects-and-infrastructure/ourinsights/reinventing-construction-through-a-productivity-revolution> (Jul. 18, 2018).

Modular Building Institute (2010). "Improving Construction Efficiency \& Productivity with Modular Construction."

<https://www.modular.org/marketing/documents/Whitepaper_ImprovingConstructionEffic iency.pdf> (Oct. 29, 2017).

Modular Building Institute (2018). "The Modular Solution to the Construction Labor Shortage." Modular Advantage, 2018Q1, 15-18. 